\title{
Migraine-Related Vertigo and Somatoform Vertigo Frequently Occur in Children and Are Often Associated
}

\author{
Thyra Langhagen $^{1}$ A. Sebastian Schroeder ${ }^{1,2}$ Nicole Rettinger ${ }^{1}$ Ingo Borggraefe ${ }^{1,2}$ Klaus Jahn ${ }^{1,3}$
}

${ }^{1}$ German Center for Vertigo and Balance Disorders (IFB), University of Munich, Germany

2 Department of Paediatric Neurology and Developmental Medicine, Hauner Children's Hospital, University of Munich, Munich, Germany

${ }^{3}$ Department of Neurology, University of Munich, Germany

Neuropediatrics 2013;44:55-58.
Address for correspondence and reprint requests Thyra Langhagen, MD, German Center for Vertigo and Balance Disorders (IFB), University of Munich, Marchioninistr. 15, 81377 Munich, Germany

(e-mail: thyra.langhagen@med.uni-muenchen.de).

\section{Abstract}

\section{Keywords}

- migraine-related vertigo

- somatoform vertigo

- benign paroxysmal vertigo in childhood
Migraine-related syndromes are a common cause of episodic vertigo and dizziness in children. Somatoform vertigo (SV) is an important cause of chronic dizziness, especially in adolescents. Our aim was to elucidate the comorbidity of migraine and SV. Three diagnostic groups were defined: migraine-related vertigo (MRV), SV, and combined migraine-related and SV (MSV). A retrospective analysis was performed on patient data (demographics, diagnosis, neuro-orthoptic and neurologic status, and results of vestibular and balance testing) from 168 patients who were presented to the German Center for Vertigo and Balance Disorders (IFB) over a 2.5-year period. Mean age of patients was $12 \pm 4$ years (range: 1.4 to 18 years). The most frequent diagnosis was MRV (28\%), followed by MSV (19\%) and SV (14\%). MSV occurred most frequently in adolescent girls (25\%). MRV was the most common cause of dizziness in our cohort. MSV ranked second overall but ranked first in adolescent girls, followed by isolated SV. SV was most prevalent in adolescent girls. MRV, MSV, and SV account for about $60 \%$ of diagnoses established in our tertiary referral center. Competent care of childhood migraine should include skill in detecting both the clinical symptoms of vertigo and overlapping somatoform symptoms.

\section{Introduction}

Of the children and adolescents who present with dizziness, 35 to $60 \%$ also have headache, which can precede, follow, or occur simultaneously with vestibular symptoms. ${ }^{1,2}$ Migrainerelated syndromes (such as benign paroxysmal vertigo in childhood [BPVC] and vestibular migraine [VM]) are the most common cause of episodic vertigo and dizziness in children. ${ }^{3-7}$ The term VM was established on the basis of clinical observation and epidemiologic data from adults. ${ }^{8,9}$ The diagnostic criteria for VM proposed by Neuhauser and colleagues ${ }^{10}$ were recently validated. ${ }^{9,11}$ VM is characterized by episodic attacks of rotatory or to-and-fro vertigo lasting minutes to hours, followed or accompanied by headache and sensitivity to light and noise. However, about $30 \%$ of adult VM patients lack such associated headaches. ${ }^{8}$ Discrete ocular motor signs (i.e., saccadic pursuit, downbeat/upbeat nystagmus, gaze-evoked nystagmus) are commonly observed in the intervals between attacks. ${ }^{8}$ The presentation of VM varies in children; it can be atypical, particularly in patients younger than 10 years of age.

BPVC is the most common cause of episodic vertigo in children between the ages of 2 and 5 years. First described in $1964,{ }^{12}$ it is characterized by short attacks of vertigo or received

September 27, 2012 accepted after revision November 12, 2012 published online January 10, 2013
Issue Theme Headaches in Childhood and Adolescence; Guest Editor, Florian Heinen, MD.
DOI http://dx.doi.org/ 10.1055/s-0032-1333433. ISSN 0174-304X. (c) 2013 Georg Thieme Verlag KG Stuttgart · New York 
dizziness (seconds to minutes) and is often associated with nystagmus and postural imbalance. BPVC is associated with a positive family history of migraine and the development of typical migraine later in life.,13 The classification of migraine-related vertigo (MRV) has not yet been well standardized in childhood and adolescence.

The term somatoform vertigo (SV) was originally proposed to describe adult patients. ${ }^{14} \mathrm{SV}$ is defined as a psychosomatic disorder associated with different psychiatric comorbidities (anxiety disorders, depressive, dissociative, and somatoform disorders). ${ }^{14}$ Adult patients with primary SV most often present with phobic postural vertigo in particular situations; it can be distinguished from secondary SV, which develops after different vestibular disorders. So far, SV, functional, or so-called psychogenic vertigo syndromes have not been systematically evaluated in children, but observational data suggest they are important causes of vertigo and dizziness in childhood, ${ }^{3,4,7}$ particularly in adolescence. ${ }^{4}$

VM commonly occurs in association with SV in adults. 9,10 Although the neurologic basis of the comorbidity of balance disorders, anxiety disorders, and migraine was recently suggested, ${ }^{15,16}$ the clinical problems of differentiating MRV, SV, and its combination (MSV) with regard to treatment and clinical course have not yet been resolved. Here, we present first-hand cohort data from a German tertiary vertigo and dizziness center in Munich on the comorbidity of VM and SV in children and adolescents for future research.

\section{Methods}

A retrospective analysis was performed on the data of children and adolescents consecutively referred to the German Center for Vertigo and Balance Disorders (IFB) from November 2009 to April 2012 (29 months). The database included patient demographics, final diagnosis, neuro-orthoptic and neurologic status, and vestibular (head-impulse test, caloric irrigation with video-oculography) and balance testing data (static posturography). Recently proposed criteria for the diagnosis of VM (without distinguishing between definitive and probable VM) ${ }^{11}$ as well as applied migraine diagnostic criteria adapted for children ${ }^{17}$ were used. To diagnose BPVC, the criteria of the International Classification of Headache Disorders of 2004 were applied. ${ }^{17}$ SV was diagnosed on the basis of criteria suggested by Eckhardt-Henn et al. ${ }^{14}$ Three groups were defined: MRV, fulfilling the criteria of VM and/or BPVC; SV, fulfilling the criteria of SV; and MSV, fulfilling both the criteria of VM and/or BPVC and the criteria of SV. The Fischer exact test was used to test for associations between diagnosis groups and laboratory test results.

\section{Results}

Of a total 168 patients, 21 patients were not included in the study because they did not report any dizziness or vertigo. The mean age of the remaining 147 patients was $12 \pm 4$ years (range: 1.4 to 18 years; 82 female).

The most frequent diagnoses were MRV (28\%), MSV (19\%), SV (14\%), and peripheral vertigo (14\%) (benign paroxysmal

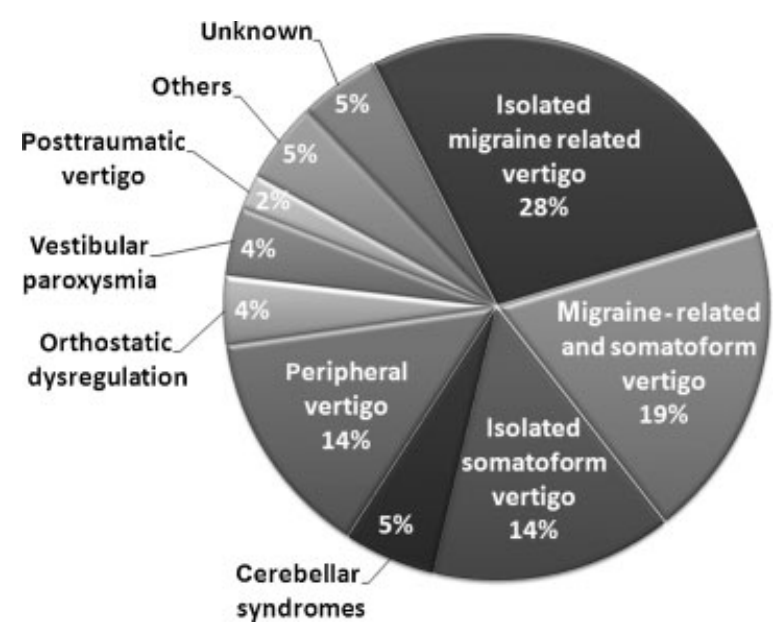

Fig. 1 Relative frequency (\%) of vertigo subtypes in 147 children and adolescents (ages 1 to 18 years) who had consulted the specialized outpatient clinic for dizziness, vertigo, or balance disorders over a period of 29 months.

positioning vertigo, Ménière's disease, vestibular neuritis, labyrinthitis, bilateral vestibulopathy). The remaining percentage (25\%) included cerebellar syndromes, orthostatic dysregulation, vestibular paroxysmia, posttraumatic vertigo, other, and unknown causes (- Fig. 1). None of our patients with BPVC $(n=6)$ had combined SV and were therefore included in the MRV group. The frequency of the three main diagnostic groups (MRV, MSV, and SV) is represented in - Fig. 2 by gender and age. MRV was the most common cause of vertigo in boys and girls under 12 years of age; the most frequent diagnosis in girls over 12 years of age was MSV. SV was diagnosed in $14 \%$ of our patients and was the second most frequent diagnosis in girls older than 12 years (21\%).

Six of 21 patients (28\%) in the SV group fulfilled the criteria of an underlying psychiatric disorder (3 with anxiety disorders, 2 with mixed anxiety-depressive disorders, 1 with a dissociative disorder). Three patients were diagnosed to have secondary SV after severe orthostatic dysregulation.

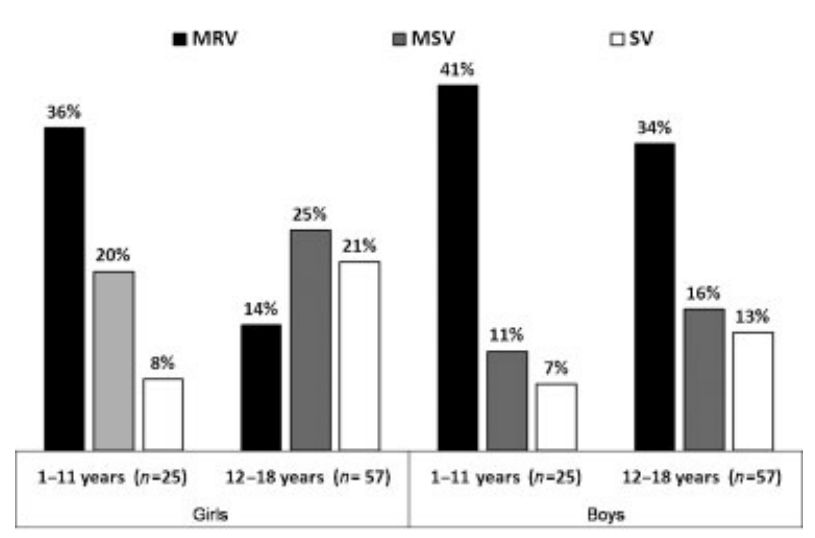

Fig. 2 Relative frequency of the three most common diagnostic groups for boys and girls in different age groups ( 1 to 11 years and 12 to 18 years). MRV, migraine-related vertigo; MSV, combined migraine-related and somatoform vertigo; SV, somatoform vertigo. 
Caloric testing was pathologic (i.e., side-to-side differences $>25 \%$ and/or peak slow phase velocity $<5$ degrees/s) in $30 \%$ $(n=72)$ and posturography was abnormal (i.e., increased postural sway at baseline with paradoxical improvement with eyes closed) in $43 \%(n=65)$ of patients; there were no significant differences between groups. Subtle abnormalities of ocular motor signs (i.e., saccadic pursuit) were more evident in MRV patients (29\%) than in MSV patients (18\%) or SV patients (14\%); however, these differences were not statistically significant.

\section{Discussion}

As a tertiary care referral center, the German Center for Vertigo and Balance Disorders (IFB) generally sees patients with chronic and recurrent syndromes, whereas patients with acute vertigo (i.e., otitis media, posttraumatic vertigo, vestibular neuritis/labyrinthitis, and neoplasia) are usually diagnosed and treated by their primary physicians. Here we focus on migraine and SV as the most common causes of chronic dizziness.

MRV is the most frequent diagnosis in our patient population. If patients with MRV and MSV are combined, migraine accounts for almost $50 \%$ of all patients treated. This high prevalence of migraine agrees with the relevant literature. A review of more than 2,000 children with dizziness reported that $25 \%$ had a "migrainous equivalent" and 20\% had BPVC. ${ }^{5}$ MRV is also frequent in smaller retrospective series, ranging from 19 to $38 \%{ }^{1,3,4,6}$ The variation in prevalence reflects differences in the diagnostic spectrum depending on the discipline (neurology, ear-nose-throat, or pediatric service) as well as the unclear diagnostic criteria. A consequence is that treatment recommendations have not been established.

$\mathrm{SV}$ is the second most common diagnosis in our patient population. If we include MSV patients, a third of our patients (33\%) present with a component of SV. SV is even more common in pubescent girls older than 12 years of age (46\%). This shift toward SV in adolescents is supported by the literature. "Extravestibular disorders" (e.g., panic and anxiety disorders) were revealed to be the most frequent cause of dizziness (24\%) in teenagers (ages 14 to 18 years). ${ }^{4}$ In a retrospective study, psychogenic vertigo was diagnosed in $22 \%$ of patients under 18 years of age experiencing dizziness. ${ }^{3}$ So far, there are almost no data available on the clinical course and treatment options for SV in children.

Discrete central ocular motor signs (i.e., horizontal saccadic pursuit) were observed most often in MRV, less commonly in MSV, and least of all in SV. Statistical significance was not reached in our cohort, probably due to the small sample size. Vertical saccadic pursuit was not considered pathologic, because vertical smooth pursuit matures in late adolescence, whereas horizontal smooth pursuit functions perfectly at the age of 8 years. Smooth pursuit eye movement abnormalities (tested by electronystagmography) were reported to be more frequent in children (45\%) than in adolescents (26\%) with vestibular disorders. ${ }^{4}$ The immaturity of the ocular motor system and/or more central vestibular dysfunction ( $26 \%$ in childhood and $18 \%$ in adolescence) may explain these findings. We were not able to compare our data with those of this study, because it did not report any correlation with the different diagnoses or whether vertical saccadic pursuit was considered pathologic. Minor central ocular motor signs were found to occur in the attack-free interval in more than $60 \%$ of adult patients with VM. ${ }^{8}$ In contrast, we observed central ocular motor signs less often despite using the same diagnostic workup as reported previously. $^{8}$ This is in part explained by the fact that we did not consider isolated vertical saccadic pursuit to be a pathologic finding in our patients, although it is the most frequent central ocular motor sign described in adult patients (48\%). ${ }^{8}$

\section{Overlap between Vestibular Migraine and Somatoform Vertigo}

Comorbidity of balance disorders, anxiety, and migrainerelated symptoms has been described in adults. ${ }^{18}$ This association might reflect subtle functional alterations in the brain of migraineurs as well as interoceptive and cognitive adaptations. The network of afferent loops in brainstem and cortex for sensoriomotoric integration shows an extensive overlap to pathways implicated in generation, perception, and regulation of emotions and affective states. ${ }^{16}$

A comorbidity of MRV and SV, which we called MSV, was found in $19 \%$ of patients in our cohort. This association was most frequent in girls older than 12 years of age. Forty-four percent of the patients with MRV also had SV. Several studies have reported on the comorbidity of balance disorders, anxiety disorders, and migraine in adults. ${ }^{15,16,18,19}$ In 2004 a name for the symptom complex of balance disorder, migraine, and anxiety disorder was proposed, namely migraine-anxiety related dizziness. ${ }^{20}$ Epidemiologic studies in adults provide consistent evidence for a link between migraine and vestibular symptoms. ${ }^{9}$ In adults, the prevalence of migraine was 1.6 times higher in 200 dizziness clinic patients compared with 200 age- and sex-matched control subjects. ${ }^{10}$ It was disclosed that $65 \%$ of adult patients with VM showed manifest comorbid psychological disorders (anxiety and phobic disorders and depression). ${ }^{18}$ In children, no similar studies have been published to date.

Ménière's disease is another organic vestibular disorder in adults often presenting with SV. ${ }^{14,18,19}$ In psychometric testing, these patients and patients with VM were found to have high values for anxiety and depression. ${ }^{19} \mathrm{~A}$ psychological assessment of children and adolescents with BPVC $(n=21)$ or migraine $(n=20)$ and control subjects $(n=19$; ages 4 to 15 years) using the Child Behavior Checklist, the Children's Depression Inventory, and the Multidimensional Anxiety Scale for Children found that BPVC and VM patients had higher Child Behavior Checklist scores (total, internalizing, and externalizing) as well as higher Children's Depression Inventory and Multidimensional Anxiety Scale for Children scores than control subjects. ${ }^{21}$ The unpredictability of vertigo attacks appears to be a significant factor for the development of anticipation anxiety in adult patients with migraine. ${ }^{18}$ Adult patients perceive a sensation of panic-like anxiety during the attacks. ${ }^{18}$ The children in our cohort often 
described panic-like anxiety during attacks in a similar way. This might subsequently lead to the development of a somatoform dizziness syndrome. It is important in the diagnosis of such patients not to overlook migraine attacks behind the somatoform syndromes. ${ }^{18}$ In view of the fact that MSV is common in children, future studies should focus on these patients to establish diagnostic criteria and establish treatment strategies to prevent chronification.

\section{Conclusions}

In the presented cohort, MRV was the most common cause of vertigo and dizziness in children and adolescents. Combined MSV was the second most frequent diagnosis, followed by isolated SV and peripheral vertigo syndromes. SV becomes more prevalent in adolescent girls. MRV and SV accounted for about $60 \%$ of all chronic and recurrent vertigo diagnoses. The pathophysiologic basis of the substantial overlap between MRV and SV is still insufficiently understood but has to be elucidated to establish adequate treatment strategies. The diagnostic and therapeutic competence for migraine management should include the competence for vertigo and dizziness as well as for somatoform disorders and their psychiatric comorbidities. Treatment of migraine has to deal with pain, vertigo, and somatoform overlapping, a complex interaction that must be elucidated in further studies.

\section{Acknowledgments}

This project was supported by funds from the German Federal Ministry of Education and Research (BMBF) under the grant code IFB 01 EO 0901. We thank Judy Benson for editorial assistance and Otmar Bayer, MD, MPH (Clinical Study Center of IFB), for help with statistical analysis.

\section{References}

1 Balatsouras DG, Kaberos A, Assimakopoulos D, Katotomichelakis M, Economou NC, Korres SG. Etiology of vertigo in children. Int J Pediatr Otorhinolaryngol 2007;71(3):487-494

2 Humphriss RL, Hall AJ. Dizziness in 10 year old children: an epidemiological study. Int J Pediatr Otorhinolaryngol 2011; 75(3):395-400
3 Gruber M, Cohen-Kerem R, Kaminer M, Shupak A. Vertigo in children and adolescents: characteristics and outcome. Sci World J 2012;109624 doi: 10.1100/2012/109624

4 Szirmai A. Vestibular disorders in childhood and adolescents. Eur Arch Otorhinolaryngol 2010;267(11):1801-1804

5 Wiener-Vacher SR. Vestibular disorders in children. Int J Audiol 2008;47(9):578-583

6 Niemensivu R, Kentala E, Wiener-Vacher S, Pyykkö I. Evaluation of vertiginous children. Eur Arch Otorhinolaryngol 2007;264(10): 1129-1135

7 Jahn K, Langhagen T, Schroeder AS, Heinen F. Vertigo and dizziness in childhood-update on diagnosis and treatment. Neuropediatrics 2011;42(4):129-134

8 Dieterich M, Brandt T. Episodic vertigo related to migraine (90 cases): vestibular migraine? J Neurol 1999;246(10):883-892

9 von Brevern M, Neuhauser H. Epidemiological evidence for a link between vertigo and migraine. J Vestib Res 2011;21(6):299-304

10 Neuhauser H, Leopold M, von Brevern M, Arnold G, Lempert T. The interrelations of migraine, vertigo, and migrainous vertigo. Neurology 2001;56(4):436-441

11 Radtke A, Neuhauser H, von Brevern M, Hottenrott T, Lempert T. Vestibular migraine-validity of clinical diagnostic criteria. Cephalalgia 2011;31(8):906-913

12 Basser LS. Benign paroxysmal vertigo of childhood. (A variety of vestibular neuronitis). Brain 1964;87:141-152

13 Lindskog U, Odkvist L, Noaksson L, Wallquist J. Benign paroxysmal vertigo in childhood: a long-term follow-up. Headache 1999; 39(1):33-37

14 Eckhardt-Henn A, Tschan R, Best C, Dieterich M. [Somatoform vertigo syndrome]. Nervenarzt 2009;80(8):909-917

15 Balaban CD. Migraine, vertigo and migrainous vertigo: links between vestibular and pain mechanisms. J Vestib Res 2011; 21(6):315-321

16 Balaban CD, Jacob RG, Furman JM. Neurologic bases for comorbidity of balance disorders, anxiety disorders and migraine: neurotherapeutic implications. Exp Rev Neurother 2011;11(3):379-394

17 Headache Classification Subcommittee of the International Headache Society. The International Classification of Headache Disorders. 2nd edition. Cephalalgia 2004;24(Suppl 1):9-160

18 Eckhardt-Henn A, Best C, Bense S, et al. Psychiatric comorbidity in different organic vertigo syndromes. J Neurol 2008;255(3): 420-428

19 Best C, Eckhardt-Henn A, Diener G, Bense S, Breuer P, Dieterich M. Interaction of somatoform and vestibular disorders. J Neurol Neurosurg Psychiatry 2006;77(5):658-664

20 Furman JM, Balaban CD, Jacob RG, Marcus DA. Migraine-anxiety related dizziness (MARD): a new disorder? J Neurol Neurosurg Psychiatry 2005;76(1):1-8

21 Reale L, Guarnera M, Grillo C, Maiolino L, Ruta L, Mazzone L. Psychological assessment in children and adolescents with benign paroxysmal vertigo. Brain Dev 2011;33(2):125-130 